\title{
Perioperative anaphylaxis to chlorhexidine: Crucial role of in-vitro testing
}

\author{
Alessandro Buonomo, ${ }^{1}$ Arianna Aruanno, ${ }^{1}$ Valter Perilli, ${ }^{2}$ Angela Rizzi, ${ }^{2}$ Manuela Ferraironi, ${ }^{3}$ Eleonora Nucera ${ }^{1}$
}

\begin{abstract}
Background: Chlorhexidine is a synthetic biguanide with a broad antibacterial activity and has become an important cause of perioperative anaphylaxis.
\end{abstract}

Objective: Reactions due to chlorhexidine allergy are usually IgE-mediated. The aim of this report is to demonstrate utility of laboratory in-vitro testing for diagnosis.

Methods: We report the case of a 36-year old man who experienced severe anaphylaxis during general anesthesia. He underwent skin tests, specific detection of specific IgE to chlorhexidine and basophil activation test (BAT).

Results: Skin tests gave false positive results due to dermographism. So, on the basis of a clinical reaction to chlorhexidine and positive tests for IgE to chlorexidine and BAT, we assessed the diagnosis of chlorhexidine allergy.

Conclusion: Physicians should be aware of the role of chlorhexidine in the etiology of perioperative anaphylaxis. In vitro testing such specific IgE and BAT are useful in patient with suspected chlorexidine allergy and limitation to perform skin tests.

Key words: Chlorhexidine, basophil activation test, drug allergy, perioperative anaphylaxis, tryptase

\section{From:}

${ }^{1}$ Allergy Unit, Fondazione Policlinico Universitario Agostino Gemelli IRCCS, Rome, Italy

${ }^{2}$ Department of Anesthesiology, Fondazione Policlinico Universitario Agostino Gemelli IRCCS, Rome, Italy

${ }^{3}$ Department of Microbiology, Fondazione Policlinico Universitario Agostino Gemelli IRCCS, Rome, Italy

\section{Introduction}

Chlorhexidine is a synthetic biguanide with a broad antibacterial activity against Gram-positive and Gram-negative bacteria, yeasts and viruses. Chlorhexidine is widely used and can be found in many products in health care setting (skin disinfectants, lubricant gels, impregnated in central venous catheters, gels for endoscopic and gynecological procedures, mouthwash, etc.). It is also used as preservative in some cosmetic products. ${ }^{1,2}$

Perioperative anaphylaxis is a rapid onset life-threatening hypersensitivity reaction usually caused by drugs used during anesthesia and surgery. Its frequency can be estimated between 1 in 4500 and 1 in 25000. ${ }^{1}$ Neuromuscular blocking agents (NMBA) and antibiotics and at lesser extent latex, opioids and other drugs may be involved.

\section{Corresponding author:}

Alessandro Buonomo

Allergy Unit, Fondazione Policlinico Universitario Agostino Gemelli IRCCS,

Largo Gemelli, 8, 00168 Rome, Italy

E-mail: alessandro.buonomo@policlinicogemelli.it

Despite its safety profile, chlorhexidine has become an important cause of perioperative anaphylaxis being responsible of about $7.7-9.6 \%$ of reported cases. ${ }^{3}$ Reactions due to chlorhexidine allergy are IgE-mediated and often severe (grades 3 or 4). Hypotension is the most common reported symptom, but urticaria, bronchospasm and angioedema are also reported. ${ }^{4}$

The correct diagnosis is crucial in case of perioperative anaphylaxis and the following test are actually available to assess the diagnosis of chlorhexidine allergy: skin prick test; intradermal test; detection of specific IgE; histamine release test; basophil activation test (BAT). ${ }^{4,5}$ 


\section{Report of a case}

A 36-year old man of Indian origin required surgery for a colorectal cancer and was scheduled for a laparoscopic colorectal resection. Firstly he was given cefazolin $2 \mathrm{~g}$, ranitidine $50 \mathrm{mg}$ and dexamethasone $4 \mathrm{mg}$ and then anesthesia was induced with fentanyl $40 \mathrm{mcg}$, lidocaine $40 \mathrm{mg}$, midazolam 1 $\mathrm{mg}$, propofol $120 \mathrm{mg}$ and rocuronium $30 \mathrm{mg}$. He was intubated and underwent catheterization of the radial artery. Skin was previously disinfected with chlorhexidine $2 \%$ and alcohol $70^{\circ}$. After 30 seconds from catheterization, he became acutely hypotensive $(50 / 20 \mathrm{~mm} \mathrm{Hg})$ with no sign of urticaria and/or bronchospasm. He was immediately treated with norepinephrine, methylprednisolone, clorphenamine and hydrocortisone. When hypotension resolved, surgery was completed with no side-effects.

Tryptase (ImmunoCAP, Phadia AB, Uppsala, Sweden) after the event was $49.5 \mathrm{mcg} / \mathrm{L}$ (normal range $<9.4 \mathrm{mcg} / \mathrm{L}$ ) confirming the diagnosis of anaphylaxis.

One month after the event the patient was referred to our Allergy unit. We tried to perform skin tests with the involved drugs but the patient showed an important dermographism (skin prick and intradermal tests with saline gave a positive wheal and flare reaction).

\section{We then performed the following test:}

- Specific IgE for chlorhexidine, ampicillin, penicilloyl G, penicilloyl V, amoxicillin, cephaclor, latex, succinylcholine, morphine and pholcodine (ImmunoCAP): criterion for positivity $\mathrm{IgE}>0.35 \mathrm{kU}_{\mathrm{A}} / \mathrm{L}$;

BAT (Flow-Cast, Bühlmann Laboratories AG, Schönenbuch, Switzerland) with propofol, cephazolin, rocuronium, lidocaine and chlorhexidine. Chlorhexidine used to stimulate basophils was obtained from Neoxinal $(100 \mathrm{~mL}$ of product contain $0.5 \mathrm{~g}$ of chlorhexidine and $70 \mathrm{~g}$ of ethanol $96 \%$ ) at concentrations of $0.05 \mathrm{~g} / 100 \mathrm{~mL}, 0.005 \mathrm{~g} / 100 \mathrm{~mL}$, $0.0005 \mathrm{~g} / 100 \mathrm{~mL}$ and $0.00005 \mathrm{mg} / 100 \mathrm{~mL}$ according to literature. Basophil activation $>5 \%$ and a stimulation index $>2$ were considered a positive result. ${ }^{4}$

Specific IgE to chlorhexidine was at $3.58 \mathrm{kU}_{\mathrm{A}} / \mathrm{L}$ (total $\operatorname{IgE}$ were at $79.6 \mathrm{UI} / \mathrm{ml}$ ) and basal tryptase at $7.3 \mathrm{mcg} / \mathrm{L}$. Specific IgE to other agents gave the following results: latex 0.01 ; penicilloyl G 0.01; penicilloyl V 0.08; ampicillin 0.01; amoxicillin 0.05 ; cephaclor 0.01 ; morphine 0.01 ; succinilcholyne 0.01

The BAT showed a positive result at the concentrations of $0.005 \mathrm{~g} / 100 \mathrm{~mL}$ (40\% of activated basophils) and $0.0005 \mathrm{~g} / 100$ $\mathrm{ml}$ (37\% of activated basophils). The BAT gave a negative result for the other tested agents: cephazolin $2 \%$; propofol $1 \%$; rocuronium $3 \%$; lidocaine $1 \%$. The positive control showed an activation of $40 \%$ and the negative control of $2 \%$.

The possibility of a drug provocation test was not taken in consideration because the patient had a life-threatening reaction and in these cases this procedure is strongly contraindicated.

On the basis of a clinical reaction to chlorhexidine and two positive diagnostic test, we assessed the diagnosis of chlorhexidine allergy. ${ }^{5}$

\section{Discussion}

Chlorhexidine is an emerging and often forgotten allergen that may be responsible of severe anaphylaxis during surgery. Correct diagnosis is often delayed since several drugs (including NMBA, antibiotics, opioids, non-steroidal anti-inflammatory drugs) and latex are used during surgery and no attention is paid to chlorhexidine.

The emerging role of chlorhexidine is now well known and several reports are now available in literature. ${ }^{6-8}$

Chlorhexidine may be found in toothpastes, mouthwashes, dressings, ointments, cosmetics, acne preparations and contact lens solutions. Exposure and consequent sensitization may happen outside medical settings. ${ }^{1}$

Our patient experienced anaphylaxis, documented by the increase of serum tryptase, immediately after catheterization of the radial artery. Exposure to chlorhexidine may have occurred during this procedure since skin was previously cleaned with this agent. ${ }^{3}$

It is well known exposure via chlorhexidine-coated central venous catheters and urethral gels are frequently responsible of severe anaphylaxis, but exposure may occur also through wounds and/or surgical incisions. ${ }^{2}$ However chlorhexidine may cause anaphylaxis also after minor wound disinfection, including local anesthesia and/or artery catheterization. ${ }^{6}$

Even if most patients with chlorhexidine anaphylaxis had prior milder hypersensitivity reactions to topical chlorhexidine, highly sensitive patients may tolerate topical chlorhexidine also. ${ }^{7}$ When evaluating our patient for the first, we cleaned his skin with Neoxinal $^{\circledR}$ (chlorhexidine $0.5 \mathrm{~g} / 100 \mathrm{~mL}$ and ethanol $96 \% 70 \mathrm{~g} / 100 \mathrm{~mL}$ ) prior skin prick and intradermal tests but no systemic reactions occurred. His dermographism was probably due to contact with chlorhexidine during skin prick and intradermal tests. However the patient was told to avoid chlorexidine in the future paying particular attention to mucous contact to minimise the risk of accidental re-exposure. Since other topical antiseptics are available, desensitization to chlorexidine has never been attempted.

Diagnostic tools with good specificity and sensitivity are available for a correct diagnosis. Specific IgE have the highest sensitivity (100\%) and specificity (97\%) when compared to skin tests and histamine release test. ${ }^{5}$ Other authors found lower sensitivity (84.2\%) and specificity (93.7\%), but in this case specific IgE were compared just to skin tests. ${ }^{9}$

The BAT seem to have lower sensitivity (50\%) but the largest case series include only 6 patients. ${ }^{4}$ The BAT may be useful when diagnosis is not fully assessed by means of skin tests and specific IgE.

Skin tests and specific IgE should be considered the first step because of their wide availability. When skin tests have some kind of limitation, another in vitro test may be helpful to confirm the correct diagnosis. ${ }^{5}$

We finally underline the importance of serum tryptase since it is the only laboratory marker to confirm the diagnosis of anaphylaxis.

Allergists, surgeons and anesthesiologists should be aware of the emerging role of chlorhexidine in the etiology of perioperative anaphylaxis. Attention should be paid to milder previous reactions after chlorhexidine exposure. 
Diagnostic tools such as skin tests and specific IgE are widely available and both have good sensitivity and specificity to assess the correct diagnosis and avoid future episodes of chlorexidine anaphylaxis.

\section{References}

1. Sharp G, Green S, Rose M. Chlorhexidine-induced anaphylaxis in surgical patients: a review of the literature. ANZ J Surg. 2016;86:237-43.

2. Opstrup MS, Jemec GBE, Garvey LH. Chlorhexidine allergy: on the rise and often overlooked. Curr Allergy Asthma Rep. 2019;19:23.

3. Rose MA, Garcez T, Savic S, Garvey LH. Chlorhexidine allergy in the perioperative setting: a narrative review. Br J Anaesth. 2019;123:e95-e103.

4. Egner W, Helbert M, Sargur R, Swallow K, Harper N, Garcez T, et al. Chlorhexidine allergy in four specialist allergy centres in the United Kingdom, 2009-2013: clinical features and diagnostic tests. Clin Exp Immunol. 2017;188:380-6.
5. Opstrup MS, Malling HJ, Krøigaard M, Mosbech H, Skov PS, Poulsen LK, et al. Standardized testing with chlorhexidine in perioperative allergy - a large single evaluation. Allergy. 2014;69:1390-6.

6. Spoerl D, Jandus P, Harr T. Pitfalls and peculiarities in chlorhexidine allergy. J Allergy Clin Immunol Pract. 2016;4:991-2.

7. Postolova A, Bradley JT, Parris D, Sherr J, McGhee SA, Hernandez JD. Anaphylaxis to invasive chlorhexidine administration despite tolerance of chlorhexdine use. J Allergy Clin Immunol Pract. 2018;6:1067-9.

8. Nakonechna A, Dore P, Dixon T, Khan S, Deacock S, Holding S, Abuzakouk M. Immediate hypersensitivity to chlorhexidine is increasingly recognised in the United Kingdom. Allergol Immunopathol (Madr). 2014;42:44-9.

9. Anderson J, Rose M, Green S, Fernando SL. The utility of specific IgE testing to chlorhexidine in the investigation of perioperative adverse reactions. Ann Allergy Asthma Immnuol. 2015;114:417-32. 